\title{
La nueva configuración del sistema de partidos valenciano. Una aproximación institucionalista
}

\author{
The new configuration of the Valencian party system. \\ An institutional approach \\ María Ángeles Abellán-López / ma.abellan@umh.es \\ http://orcid.org/0000-0002-6553-0227 \\ Universidad Miguel Hernández de Elche, España \\ Gonzalo Pardo-Beneyto / gonparbe@gmail.com \\ http://orcid.org/0000-0001-7142-1740 \\ Universidad de Alicante, España
}

\begin{abstract}
The main objective of this article is to explain the evolution of the party system of the Valencian Community (Spain) between 1978 and 2015. We will start from a historical neo-institutionalist approach and a methodology focused on historical analysis (path dependence and critical junctures). It will be centered on the changes produced in political parties, in terms of the Valencian cartelization and identity and its present position. This way, three working sub-hypotheses are to be explained: H1a: parties have had a continuity dynamic that has changed due to abrupt changes in the environment; H1b: political forces have taken advantage of these events to obtain electoral revenue and; H1c: current political parties are the result of changes experienced in the period studied. This complex hypothesis opens new fields of study for future research in terms of strategy, discourse and perceptions about these organizations.
\end{abstract}

Key words: party system, institutionalism, cartelization, identity, Valencian community.

Resumen: El objetivo del artículo es explicar la evolución del sistema de partidos de la Comunitat Valenciana (España) entre 1978 y 2015. Se parte de un enfoque neoinstitucionalista y de una metodología centrada en el análisis histórico (path dependence y critical junctures) de los cambios producidos en los partidos políticos, en cuanto a la cartelización y la identidad valenciana, y su caracterización actual. Así, se han generado tres subhipótesis de trabajo: H1a: los partidos han tenido una dinámica continuista que ha variado por cambios bruscos del entorno; $\mathrm{H} 1 \mathrm{~b}$ : las fuerzas políticas han aprovechado dichos acontecimientos para obtener rédito electoral; y $\mathrm{H} 1 \mathrm{c}$ : los actuales partidos políticos son fruto de los cambios experimentados en el periodo estudiado. Esta hipótesis compleja abre nuevos campos de estudio para futuras investigaciones en cuanto a estrategia, discurso y percepciones sobre estas organizaciones.

Palabras clave: sistema de partidos, institucionalismo, cartelización, identidad, Comunitat Valenciana. 


\section{Introducción}

El presente trabajo tiene como principales objetivos ofrecer una panorámica del sistema de partidos valencianos, así como la generación y respuesta de hipótesis, mediante el análisis y la interpretación de algunos de los cambios experimentados en los partidos políticos de la Comunitat Valenciana (España). Para ello, nos centraremos en parte de las variables propias del partido cártel y en la evolución del componente identitario de esta región española. Nuestro propósito será responder a la hipótesis de partida de forma exploratoria:

H1a. Los partidos valencianos han tenido una dinámica continuista en cuanto a resultados se refiere que solo se ha visto quebrada por acontecimientos fortuitos o cambios sociales bruscos.

H1b. Cuando ha habido un cambio de esta naturaleza, las fuerzas politicas lo han aprovechado para obtener rédito electoral, lo cual ha llevado al sistema de partidos a sufrir cambios.

H1c. Los partidos actuales son fruto de los cambios experimentados durante el periodo histórico estudiado.

Con base en un enfoque neoinstitucionalista, utilizaremos una metodología sustentada en el análisis evolutivo del fenómeno examinado. Dadas las limitaciones formales del artículo, se establecerá un nivel de investigación macro, cuyo objeto de estudio son las relaciones interpartidistas en las que los partidos políticos, como actores privilegiados, cambian su estrategia a lo largo del tiempo, como respuesta a las modificaciones del entorno.

La idea subyacente es que el sistema de partidos valenciano es estable porque está inmerso en una trayectoria inercial que lo condiciona y que solo cambia si se produce una disrupción, fruto de un acontecimiento o cambios fortuitos en variables básicas de la sociedad (Mahoney, 2000; Peters, 2003; Pierson, 2004).

Este trabajo actualiza y complementa otros que ya analizaron una parte del sistema de partidos o su totalidad, como los de: Bodoque (2009), Català (2014), Franch (1995, 1996 y 1998), Franch y Hernández (2005), Franch y Martín-Cubas (1999), Martín-Cubas (2007 y 2015), Martínez-Sospedra (1996 y 1999), Oñate (2013) y Sanmartín (2010), sobre los elementos constitutivos y genuinos del sistema electoral, el sistema de partidos y el comportamiento electoral de los votantes de la Comunitat Valenciana.

Pero, al mismo tiempo, el estudio de la evolución del sistema de partidos valenciano es un buen punto de partida, y representativo, para entender las modificaciones que se están produciendo a nivel político y social en el Estado 
español. La Comunitat Valenciana es una entidad subestatal que se encuentra en una posición geográfica periférica respecto a Madrid, como capital del Estado, y que, al mismo tiempo, comparte rasgos culturales y lingüísticos con Cataluña, que, como veremos, han marcado la construcción institucional y la del propio sistema de partidos valenciano.

Este ha experimentado una serie de cambios, como el paso del tradicional turnismo de largos periodos entre el Partido Socialista Obrero Español (PSOE) (1983-1995) y el Partido Popular (PP) (1995-2015) en el gobierno, con cotas de concentración de voto entre el 80 y el $90 \%$, a un escenario donde dicha concentración está en torno al 50\% (Pardo y Abellán, 2016).

El presente artículo se estructura de la siguiente manera: en primer lugar, se explicará el enfoque del neoinstitucionalismo histórico y los conceptos de path dependence y critical junctures, marco teórico del que partimos. Después se definirá la idea de cartelización en los partidos políticos y la generación de las estrategias poscártel. Luego se abordarán las fases y lineamientos estratégicos, con especial énfasis en el conflicto identitario valenciano. En seguida se discutirán de forma comparada las principales características de los actores del sistema de partidos valenciano, mediante sus estrategias cártel/poscártel y su posicionamiento respecto a la cuestión identitaria. Por último, se esbozarán las principales conclusiones en las que se generará una hipótesis compleja y se plantearán las posibles líneas futuras de investigación.

\section{Marco teórico}

El neoinstitucionalismo es un enfoque de la ciencia política que se gesta como una reacción tanto a los estudios que se centraban en el individualismo metodológico como a los que procedían del institucionalismo clásico caracterizado por: a) una exacerbada juridicidad, b) el formalismo, c) la escasa pluralidad en el tratamiento de las instituciones que conforman el sistema político de un país, y d) el recurso a la historia poco sofisticado.

El fruto de esta reacción produce un interés por los comportamientos de los individuos en una sociedad dada, cómo se relacionan con los aspectos culturales y cómo se desempeñan las instituciones y organizaciones en los fenómenos sociopolíticos.

Con este enfoque coral se pretende entender los fenómenos políticos desde una nueva perspectiva e incide en los aspectos institucionales, así como en el comportamiento de los actores para explicar el proceso político (Easton, 1997; Immergut, 1998; Pierson, 2004; Mahoney, 2000; Peters, 2000). 
El enfoque neoinstitiucionalista admite varias versiones, y una de ellas es la conocida como neoinstitucionalismo histórico que, como indica su nombre, se centra en los aspectos históricos y sus efectos en las instituciones. El término institución para estos estudiosos hace referencia a los aspectos formales y a las normas y leyes que rigen una sociedad concreta (Peters, 2003).

La historia es importante y las decisiones que se toman a la hora de diseñar las instituciones marcan una inercia que sigue teniendo efectos a largo plazo y de la cual no es fácil revertir los acontecimientos. Por esta razón, Steinmo (2013) sostiene que: a) un entorno político específico es el que determina los fenómenos políticos; b) la historia es un elemento de aprendizaje; y c) condiciona las expectativas de los individuos. Estos tres aspectos se vinculan a dos conceptos capitales de este enfoque: la path dependence y las critical junctures.

La path dependence, traducida como inercia institucional, se refiere a la forma como se analiza el problema de investigación y a cómo se construyen las causas que determinan los fenómenos sociales a través de cadenas de fenómenos contingentes; es decir, explica el desarrollo de una secuencia de eventos en un relato histórico y cómo, cuando se produce un acontecimiento importante, el curso de los acontecimientos se verá alterado para siempre (Landman, 2011).

Estas relaciones entre eventos dan lugar a dos fenómenos complementarios: a) los efectos de refuerzo en los que la dinámica creada se perpetúa en el tiempo al crearse incentivos, para que así sea en un punto de equilibrio y b) la reactividad que se produce por un fenómeno acaecido que contradice la inercia propia de las instituciones y rompe el punto de equilibrio anterior (Mahoney, 2000; Pierson, 2004).

La dinámica de las critical junctures introduce el cambio institucional en la path dependence formando un modelo dual, en el cual se hallan largos periodos de inercia con elementos de cambio producidos por fenómenos fortuitos, propios del entorno donde se encuentran las instituciones. Dichos eventos son críticos porque rompen la inercia con la que una institución actuaba para ocasionar efectos contrarios a la misma (Capoccia y Kelemen, 2007).

Junto a los dos conceptos capitales mencionados, hemos de añadir la vertiente estratégica de la toma de decisiones. En última instancia, las decisiones estratégicas de los actores se centran en la supervivencia, tanto del responsable de llevarla a cabo, como de aquellos que apoyan el cambio de statu quo o su mantenimiento (DiMaggio, 1988; Mahoney y Thelen, 2010; Oliver, 1988; Pfeffer y Salancik, 1978; Thelen, 2003). 
Tal como veremos más adelante, las relaciones estratégicas se producen en los partidos políticos, como actores fundamentales del sistema de partidos valenciano, y cuando se producen actos fortuitos en el entorno, sus organizaciones reaccionan en pro de la mejora de la competitividad de su formación política.

Una vez planteado el enfoque para analizar los procesos de cambio en el sistema de partidos valenciano y de sus actores, vamos a centrarnos en el fenómeno de la cartelización de los partidos políticos y el sentimiento identitario - con sus controversias y complejidades-, para conocer su dinámica en la Comunitat Valenciana.

\section{Las estrategias cártel y poscártel en el sistema de partidos valenciano}

Los partidos políticos constituyen piezas clave del sistema representativo, pues, como intermediarios, son los protagonistas indiscutibles de y para la gobernabilidad. No sólo suministran líderes y candidatos que colocan en las instituciones, sino que transforman la sociedad mediante intervenciones contenidas en sus programas políticos, herramientas fundamentales de identificación ideológica.

Como es sabido, dichas organizaciones han sufrido una serie de cambios en sus cometidos y organización, pasando de un partido de cuadros a uno de masas, para, posteriormente, transformarse en partidos "atrapalotodo" (Kirchheimer, 1966). Esta expresión caracteriza a los partidos que buscan ampliar al máximo su base electoral interclasista, tienden hacia el pragmatismo político con la adopción de programas políticos flexibles y con menor ortodoxia ideológica para captar el mayor número de votos. En esta trayectoria evolutiva se produce una adaptación histórica, fruto de la separación entre sociedad civil e instituciones, que da lugar al surgimiento del partido cártel (van der Eijk, 1992; Downs, 1957; Franklin, 1992; Katz y Mair, 2012; Kirchheimer, 1966; Kitschelt, 2004).

Las características más destacables de los partidos cártel son las siguientes: a) los partidos en el gobierno definen aspectos capitales del sistema electoral, la regulación de las organizaciones partidistas, la financiación y la comunicación institucional; b) las relaciones que se producen dentro del propio partido derivan en la profesionalización de la política, la existencia de asesores profesionalizados y en una estructura de estratarquía en la que se reproducen los patrones de la organización central del partido; c) en la competencia en la arena electoral y los mensajes que se lanzan al público con un claro carácter centrípeto con el objetivo de conservar el statu quo; y d) en 
el comportamiento profesionalizado de los representantes del partido en el desempeño de cargos públicos político-administrativos (Detterbeck, 2005; Hutcheson, 2012; Kopecky y Mair, 2011; Sandri y Pauwels, 2010).

El partido cártel se fusiona con las instituciones político-administrativas, pierde la conexión con la sociedad civil y crea una relación de: dependencia, dirección y captura. El diseño del sistema electoral o la financiación de los partidos están en el origen de la creación del cártel y devienen en instrumentos que impiden que los partidos fuera del cártel puedan acceder a las estructuras político-administrativas.

Los partidos miembros del cártel se alinean en situaciones de cuasi monopolio sobre asuntos que les interesan de forma que los electores no tienen capacidad de elegir y aíslan a los partidos más pequeños con el objetivo de minimizarlos al máximo (Duverger, 1957). En cierta manera, se podría afirmar que los partidos en dicho estadio tratan de limitar el pluralismo con el fin de salvaguardar sus propios intereses como organización (Sartori, 2005).

Ante este tipo prácticas, que en España vienen reforzadas por el blindaje que se realiza de los partidos políticos en su Constitución (López-Nieto, 2010), ha aparecido una serie de estrategias partidistas encaminadas a recuperar la conexión con la sociedad civil que Yishai (2001) ha calificado como poscártel. Las estrategias poscártel consisten en el intento por parte de un partido o grupo de partidos - generalmente en la oposición- de desbancar a la formación gubernamental, asumiendo como suyo el clamor popular y las demandas de la sociedad civil con el objetivo de ganar adeptos y generar legitimidad.

Los extremos de esta estrategia poscártel son los siguientes:

1. La relación entre los partidos y la sociedad civil se articula mediante vínculos sociales, económicos e ideológicos-culturales. Las formaciones políticas asumen demandas y aspiraciones de los movimientos sociales o la defensa de causas políticas y sociales. Este apoyo puede ir más allá al convertirse en uno de tipo económico dentro de la propia campaña o con la inclusión de miembros de la sociedad civil en los puestos de responsabilidad.

2. La explotación de las fracturas en las que se divide la sociedad (Manin, 1999) -las cuales producen un sentimiento de pertenencia y se canalizan a través de la defensa de temas relacionados con el origen étnico, el género, la sexualidad o la cultura- afecta a distintos colectivos excluidos de la dinámica habitual de la vida político-social (Yishai, 2001). 
En definitiva, la estrategia poscartelización trata de minar determinados aspectos de las instituciones político-administrativas, que han sido construidos por los partidos cártel, y de reconciliarse con la sociedad civil. En la Tabla $1^{1}$ se puede encontrar las principales características de la estrategia cártel y la estrategia poscártel.

Una vez definidos ambos tipos de estrategia y sus características más importantes, vamos a centrarnos en la evolución de cada uno de los actores en las dinámicas intrapartidistas a lo largo del tiempo.

\section{La evolución del sistema de partidos valenciano}

Muchas son las variables que afectan a la formación del sistema de partidos, pero, como hemos dicho en la introducción, nos enfocaremos en el cambio de las estrategias cártel y poscártel en el periodo estudiado y pondremos un especial énfasis en la cuestión identitaria. Hemos dividido las dinámicas en cuatro periodos distintos: a) la transición valenciana y la aprobación del Estatuto de Autonomía de la Comunitat Valenciana (1978-1982); b) la construcción de la autonomía valenciana (1983-1995); c) la consolidación (1995-2015); y d) el cambio de paradigma (desde 2015 hasta la actualidad). Esta periodización será analizada a través de la path dependence y las critical junctures en el marco del neoinstitucionalismo histórico.

\section{Primer periodo (1978-1982): de la transición valenciana a la aprobación del} Estatuto de Autonomía de la Comunitat Valenciana

La transición valenciana estuvo marcada, en esta primera etapa, por dos trayectorias inerciales que afectaron a los procedimientos y arreglos para configurar el autogobierno. Por un lado, encontramos un escenario donde las fuerzas políticas con representación en las provincias de Alicante, Castellón y Valencia fueron las encargadas de pilotar la descentralización. Por otro, los instrumentos para la descentralización político-administrativa siguieron el camino marcado por el País Vasco y Cataluña, en cuanto a procedimiento y reivindicaciones se refiere (Sanz-Díaz, 1982).

Al mismo tiempo, las instituciones preautonómicas se identificaron con parte del pensamiento de Joan Fuster, lo cual reforzó el sentimiento identitario. Inicialmente tuvo el beneplácito de todas las fuerzas relevantes, a excepción de Alianza Popular. El resultado fue la aceptación de la unidad

1 Todas las tablas se encuentran al final del presente artículo, en el Anexo (Nota del editor). 
lingüística del catalán, una bandera sin una banda azul y el escudo de la Generalitat, así como la denominación de País Valencià, y se alinearon en un bloque que simpatizaba con Cataluña y el Catalanismo en distinto grado. ${ }^{2}$

La coyuntura crítica de este periodo se produjo cuando la Unión de Centro Democrático UCD, partido en el gobierno del Estado en aquel momento, cambió su postura dificultando la implementación del autogobierno valenciano y abrazó unas ideas que ya estaban siendo defendidas por Alianza Popular. El discurso de esta formación de derechas chocó frontalmente con las instituciones ejecutivas preautonómicas, y de esta colisión surgió el movimiento conocido como blaverismo. ${ }^{3}$

Los blaveros representaban un sector político encarnado por conservadores que acusaban a los autonomistas de "antivalencianos" y asumían el tradicionalismo basado en el antiguo Reino de Valencia, en la bandera coronada con franja azul, y afirmaba el valenciano como lengua propia y distinta del catalán. Estos argumentos, además, bebían de una idea del pueblo valenciano en la que este había permanecido inmaculado y puro, aunque se habían asentado diferentes pueblos mayoritarios a lo largo de la historia ${ }^{4}$ (Garcia-Oliver, 2016; Sanz-Díaz, 1982; Sanz Díaz y Felip i Sardà, 2006).

Desde entonces hasta nuestros días, este enfrentamiento identitario, lejos de languidecer, se ha cronificado en la escena pública. ${ }^{5}$ En definitiva, el blaverismo pretendió generar una legitimidad alternativa a la oficial (en la Tabla 2 se visualizan las estrategias cártel y poscártel en este periodo).

2 Los postulados de este sector de los partidos valencianos se apoyaban, en mayor o menor medida, en las ideas de Joan Fuster. Estos, en su forma plena, se justificaban en una idea germánica del término nación en el que una misma lengua, una misma cultura y, por tanto, un mismo pueblo eran la antesala de un Estado formado por dicha uniformidad. Esta concepción concuerda con la idea alemana de nación (Brubaker, 1990; Fuster, 1967).

3 El blaverismo se convirtió en un potente movimiento social que defendía la personalitat valenciana y que originalmente surgió en cuatro focos: a) las juventudes de Lo Rat Penat, una entidad cultural valencianista y referente en la promoción, defensa y difusión de la lengua y cultura valencianas; b) el Grupo d'Acció Valencianista; c) el entorno fallero; y d) la Asociación Valencia-2000, que pretendía organizar políticamente el valencianismo conservador.

4 Se decía, por parte de estos actores e "intelectuales”, que el valenciano era una lengua antigua que venía de los mozárabes e, incluso, de los íberos, contradiciendo a la corriente dominante de expertos que ha estudiado la materia (Garcia-Oliver, 2016).

5 Uno de los indicadores más representativos es la derogación de la Ley 6/2015, del 2 de abril, de Reconocimiento, Protección y Promoción de las Señas de Identidad del Pueblo Valenciano, en enero de 2016. 
El enfrentamiento entre ambas formas de entender el territorio valenciano dio lugar a una batalla de símbolos y a algún que otro altercado violento y tumultuoso que se encuadró en la conocida "Batalla de Valencia". Si bien el Estatuto de Autonomía valenciano fue aceptado por los demócratas, los alineamientos en torno a la lengua, la bandera y a la denominación del territorio estaban lejos de un cierto consenso y se caracterizaron por una gran beligerancia en varios frentes.

Dicho periodo acaba con la formulación del Estatut de Benicàssim, que se convertirá posteriormente en el Estatuto de Madrid al pasar por alto el acuerdo entre el Partido Socialista del País Valenciano (PSPV-PSOE) y la UCD sobre los símbolos estatutarios, y ceder su conformación a los parámetros de la derecha política (Sanz-Díaz, 1982). El Estatuto de Autonomía también marca un antes y un después en cuanto a la identidad, habiendo partidos que optan por tener una visión pragmática y abrazar los símbolos dispuestos por dicho texto (Vallès i Sanchis, 2000).

Este "estira y afloja" ha sufrido distintas evoluciones que marcan el alineamiento de los partidos a lo largo de los 39 años de historia de la Comunitat Valenciana como entidad política. ${ }^{6}$ Dicha división en la forma de entender el territorio valenciano y su identidad supuso la conformación de tres bloques diferenciados en una primera etapa (véase Tabla 3).

\section{Segundo periodo (1983-1995): la construcción de la autonomía valenciana}

Este segundo periodo está determinado por dos path dependence. La primera inercia se materializó cuando la nueva entidad política y su concepción moderna como Comunidad Valenciana quedó marcada por la polarización entre actores y el frágil consenso al que se llegó (Mira, 2015). La ausencia de acuerdo político condicionó el diseño del proceso autonómico valenciano si tenemos en cuenta que, inicialmente, se pensó en el artículo 151 de la Constitución Española (CE) para acceder a la autonomía y que, finalmente, se alcanzó mediante la vía lenta del artículo 143 y la promulgación de la LOTRAVA $^{7}$, que transfería más competencias al territorio valenciano.

Junto a esta falta apriorística de entidad propia, encontramos una segunda inercia que afectó a las elecciones autonómicas de 1983 y que reprodujo los resultados de las elecciones generales del año anterior en los que el PSOE se alzó con la victoria y se produjo la debacle de la UCD como la coalición 6 En este periodo se engloban los cuatro años de preautonomía y los 35 años de la creación de las instituciones valencianas a través de su Estatuto de Autonomía.

7 Ley Orgánica de Transferencia a la Comunidad Valenciana. 
mayoritaria en el centro derecha hasta ese momento. A esto hay que añadir la cohabitación de pequeños partidos que se sitúan a una distancia mucho mayor que estas formaciones y otros partidos que no obtienen ninguna representación (Martín-Cubas, 2015; Oñate, 2013; Reif y Schmitt, 1980).

En cuanto a los símbolos propios de la autononomía valenciana, los socialistas construyeron un consenso autonómico en torno al texto estatutario y las señas de identidad contenidos en este. La búsqueda de legitimidad por los actores políticos presentes en las instituciones materializó un discurso ambiguo hacia reivindicaciones propias del catalanismo político o el regionalismo más escorado a la derecha política (Català, 2012).

A ello hay que sumar una política económica centrada en los sectores económicos tradicionales de la economía valenciana. La coyuntura crítica en este periodo se inicia con la recesión económica de la primera mitad de la década de 1990, la aparición de múltiples casos de corrupción en la arena estatal y un creciente desgaste, fruto de una estancia en el poder prolongada en el tiempo. Se abre, pues, una etapa de beligerancia política que fue hábilmente aprovechada por el Partido Popular de la Comunidad Valenciana para acceder a las instituciones a través de un discurso modernizador, acorde con el que se producía a nivel nacional y que se traduciría a partir de 1995 en un cambio de gobierno (Català, 2012) (véase Tabla 4).

En el caso de los partidos poscártel y, más concretamente, respecto a los partidos de índole nacionalista, surge una tesis menos radical que rompe en parte con las ideas fusterianas y se centra en definir al pueblo valenciano como una entidad política que en el pasado y en la actualidad tiene autonomía propia, pero que, al mismo tiempo, comparte rasgos históricos, culturales y lingüísticos con territorios como Cataluña y las Islas Baleares. Esta manera de entender el valencianismo será interiorizada y aceptada por partidos como UPV (Unitat del Poble Valencià) como una adaptación estratégica al nuevo consenso creado con la autonomía valenciana (véase Tabla 5).

\section{Tercer periodo (1995-2015): la consolidación}

El periodo 1995-2015 empieza con una reducción en el tono de tensión ante la batalla de los símbolos. El discurso de la derecha en la cuestión identitaria mantuvo un tono de bajo perfil, al tiempo que impulsó la creación de la Academia Valenciana de la Lengua, lo cual contribuyó a su posicionamiento en una línea autonomista estatutaria y al continuismo de la inercia del periodo anterior iniciada por los socialistas. 
Las políticas seguidas por los distintos gobiernos del Partido Popular aprovecharon el periodo de bonanza de la economía española, desde la segunda mitad de la década de 1990 hasta el 2010, para desarrollar proyectos basados en grandes inversiones en equipamiento dedicado al ocio y eventos enfocados a la atracción de turistas. Este modelo se complementó con la apuesta del turismo de sol/playa y residencial que auspició el liderazgo del sector de la construcción en la actividad económica valenciana.

El predominio de gobiernos monocolor avalados por mayoría absoluta, excepto el de la legislatura 1995-1999, facilitó el despliegue espectacular del aumento del sector público, precisamente en un momento en que las teorías dominantes abogaban por una disminución y desmantelamiento de las estructuras públicas. Esta coyuntura económica devino en una inercia potente para guiar la acción de gobierno y la adopción de ulteriores decisiones.

La crisis económica, que actuó como critical juncture, acabó con el periodo de bonanza desvelando los casos de corrupción que se habían gestado durante las dos décadas de gobierno popular. Paralelamente, la sociedad valenciana asistió a un deterioro de los servicios públicos, la infrafinanciación del territorio y una percepción negativa del gobierno estatal.

Los partidos fuera de la acción de gobierno llevaron a cabo una oposición que se centró en el cuestionamiento del modelo económico, reivindicaciones de la regeneración democrática y la lucha contra la corrupción. Por consiguiente, no es de extrañar el surgimiento de alternativas partidistas e ideológicas, cuyas principales líneas programáticas se fundamentaban en la regeneración de las instituciones valencianas y las políticas públicas simbólicas (véase Tabla 6).

\section{Cuarto periodo (desde 2015 hasta la actualidad): el cambio de paradigma}

El actual periodo se caracteriza por la continuidad de las inercias anteriores, que son las que marcan la agenda política. No obstante, y dado que todavía es un periodo en conformación del juego político, resulta complicado avanzar las coyunturas críticas que se están gestando en este momento, así como la inercia producida por la path dependence.

No obstante, algunas de las tendencias heredadas y estratégicas de los periodos anteriores son las siguientes:

En primer lugar, nos encontramos en una sociedad líquida, permeada por la globalización y las interdependencias económicas y sociales en la que los grandes cambios sistémicos han afectado la manera como se perciben los fenómenos políticos y su identificación (Bauman, 2000; Berger y Luckmann, 1975; Castells, 1999). 
Desde el punto de vista de la cuestión identitaria, se produce una proliferación de formas de ver y entender la Comunitat Valenciana en las que convergen las siguientes principales vías de desarrollo de la identidad propia: a) un sentimiento de filiación española, pero con contestación anticentralista; b) un regionalismo valenciano proespañolista; c) un nacionalismo valenciano, en sentido estricto; d) un nacionalismo minoritario identificado con el ámbito de "Països Catalans", que destaca la catalanidad del País Valencià y rehúye su identificación con España; y e) un nacionalismo cívico que acepta que elementos diferenciadores como la lengua, la cultura y la historia sirven como refuerzo a un sentimiento nacional propio que se gesta en la voluntad de los individuos ${ }^{8}$ (Hernàndez i Albert, 2012; Mezquida, 2015).

En segundo lugar, encontramos que las elecciones a Cortes Valencianas de 2015 han materializado un ejecutivo de coalición, que a partir de una estrategia poscártel ha desarrollado políticas públicas opuestas a las del periodo anterior. Las líneas políticas preferenciales se han centrado en el cambio de modelo socioeconómico, la implementación de políticas simbólicas de legitimidad y transparencia, y la reivindicación y recuperación de los aspectos lingüísticos culturales propios del territorio.

Finalmente, un rasgo de la etapa actual es la proliferación de la oferta electoral con tintes regeneradores e, incluso, emancipadores. Como veremos, las formaciones políticas han aprovechado el desgaste de los principales partidos surgidos al albur de la Transición democrática, para proponer una alternativa en la que aspectos como la legitimidad, la regeneración democrática y el cambio de los usos políticos son los ejes fundamentales de su mensaje.

\section{Discusión}

Una vez expuesto el escenario de las transformaciones más relevantes que están en el origen de los cambios estratégicos de los partidos políticos durante los cuatro periodos propuestos, vamos a realizar una breve comparación de sus principales fundamentos y lineamientos programáticos, y para ello nos serviremos de los programas electorales con los cuales se presentaron a las elecciones en 2015. Concretamente, nos centraremos en los actores del sistema de partidos actual: el Partido Popular de la Comunitat Valenciana

8 Mezquida (2015) destaca que esta identidad valenciana no puede dejar de lado a la identidad predominante que, en este caso, es la española y debe integrar otros valores como el europeísmo, la defensa del medio ambiente, la regeneración democrática, el republicanismo, el feminismo o la defensa de los derechos de personas LGBTI (Lesbianas, Gays, Bisexuales, Transgénero e Intersexuales). 
(PPCV), el Partido Socialista del País Valenciano (PSPV-PSOE), Compromís, Esquerra Unida (EUPV), Ciudadanos y Podemos.

Como ya se ha anticipado, el ámbito espacial del presente estudio es la Comunitat Valenciana, pero ello no invalida que los argumentos sugeridos sean también representativos del sistema de partidos del Estado español. Esto se explica porque los partidos políticos - a excepción de Compromís- no tienen independencia respecto de las organizaciones centrales, y sus dinámicas ideológicas y estrategias partidistas reproducen los isomorfismos estatales.

Tanto el PSPV-PSOE como el PPCV son los artífices del actual sistema político-administrativo valenciano. Los socialistas constituyeron el autogobierno de la Generalitat, pactaron junto al principal partido de la oposición un sistema electoral que tiende a la concentración de voto para los principales partidos y, en definitiva, establecieron las bases para el surgimiento de partidos cártel, al crear un entramado diseñado para hacer difícil el acceso a otras fuerzas políticas. A partir de 1995 llega a la Generalitat el PPCV y no solo mantiene esta situación, sino que desarrolla una estrategia centrada en un incremento hipertrófico, sin precedentes, del sector público, el diseño de ingeniería organizativa y la colonización de las instituciones políticas administrativas.

No es de extrañar que ambos partidos defiendan el statu quo surgido de la Transición. Por un lado, el Partido Popular defiende el Estatuto y la CE, así como el bloque de constitucionalidad compuesto por la CE y los Estatutos de Autonomía de las Comunidades Autónomas. En los años de gobierno de la formación conservadora, se percibe un claro alineamiento con los aspectos materialistas centrados en la creación de empleo y de la economía para fidelizar el voto y un retroceso en las políticas públicas posmaterialistas relacionadas con el fortalecimiento del Estado de derecho y de la rendición de cuentas, la apertura de las instituciones a la ciudadanía y el fomento de la transparencia. Esta afirmación se constata en la proliferación de casos de corrupción (Della Porta, 2002; PPCV, 2015; Sanmartín, 2010).

El PSPV-PSOE ha adoptado una estrategia algo más abierta que el PPCV en su repertorio y formas. Los socialistas respetan el acuerdo de 1982 de la autonomía valenciana y defienden los aspectos políticos institucionales fundamentales del Estado español y de la planta de gobierno subestatal. Donde sí han marcado diferencias con discursos beligerantes es en aspectos de regeneración democrática y recuperación de la legitimidad, y han adoptado diversas medidas empoderadoras y de participación ciudadana (PSPVPSOE, 2015 y 2015a). 
Asimismo, proponen una reforma del Estatuto de Autonomía y de la Ley Electoral, entre otras. El giro socialista se explica por dos motivos: 1) el partido socialdemócrata ha estado 20 años fuera de las instituciones valencianas y ha necesitado una renovación de su mensaje para regresar a las instituciones públicas, y 2) la actualidad ha estado marcada por los casos de corrupción, el despilfarro y la falta de rentabilidad de las inversiones públicas, y una crisis económica galopante. En cierta manera, el PSPV-PSOE ha actuado como lo hizo el PPCV en los años noventa, prometiendo una gestión eficiente y sin casos de corrupción.

Ambos partidos tienen un alto grado de profesionalización política, y en las legislaturas sin poder gubernamental, sus cuadros se refugian en las instituciones representativas.

Desde el punto de vista organizativo interno, el PPCV todavía no realiza primarias para la elección de cargos y candidatos, y el PSPV-PSOE empezó en 2013 con un formato abierto. Respecto a la cuestión identitaria, si bien ambos son autonomistas, su tratamiento del tema difiere claramente. El PSPV-PSOE defiende las bases del Estatuto de Autonomía, lo que no obsta para realizar algún que otro guiño al nacionalismo de base fusteriana o las reformulaciones de dicha visión (Català, 2014).

En cambio, el PPCV sintoniza con el blaverismo y, en definitiva, con el centralismo español, ${ }^{9}$ sobre todo con el surgimiento del conflicto catalán y la pérdida del poder político territorial. Este discurso también lo adopta Ciudadanos con unos tintes mucho más centralistas que los populares. Por tanto, el objetivo principal del Partido Popular y Ciudadanos ha sido construir un nuevo marco conceptual para ser percibidos por los votantes como los auténticos defensores de los intereses valencianos, frente al resto de formaciones políticas que, en su opinión, representan más bien los intereses de Catalunya.

Retomando a Ciudadanos, este partido entiende que las reformas son necesarias para la mejora del funcionamiento democrático y aboga por cambios en la Ley Electoral, y el endurecimiento de los sistemas de rendición de cuentas y de la lucha contra la corrupción (Ciudadanos, 2015), compartiendo una visión anglosajona y liberal que es apoyada por gran parte de los poderes económicos españoles. No obstante, la formación de Albert Rivera ${ }^{10}$ cree en los consensos básicos surgidos de la Transición y del Estatuto, aunque

9 Por ejemplo: a) a finales de su mandato, en 2015, promulgaron una Ley de Señas de Identidad en contra del consenso creado con la aprobación del Estatuto, y b) se oponen a una política de fomento y uso del valenciano y acusan al ejecutivo de izquierdas de procatalán y antivalenciano.

10 Líder y fundador de Ciudadanos. 
no cierra la puerta a modificaciones relacionadas con la mejora del funcionamiento del Estado de las Autonomías, protegiendo la igualdad y la equidad entre españoles.

Tanto Ciudadanos como EUPV, Compromís y Podemos celebran primarias para la elección de candidatos a las elecciones. Estos tres últimos partidos, posicionados a la izquierda del espectro ideológico, comparten un amplio programa transformador de emancipación política que recoge las tendencias del $15 \mathrm{M}$, una tematización de sus repertorios y varias estrategias poscártel.

Compromís, formación de izquierda y nacionalista, aprovechó la debilidad del PPCV para denunciar casos de corrupción durante toda la legislatura y utilizó las redes sociales para visibilizar su discurso en los medios de comunicación de masas. Una estrategia similar fue la de EUPV denunciando ante el juzgado el Caso Taula y la mala praxis que se produjo en la Diputación de València.

EUPV se ha centrado en la reivindicación de cambios en el sistema político-administrativo, encaminados a buscar la participación de la ciudadanía a través de la democracia directa y la deliberación, la ampliación del sufragio a los mayores de 16 años (Acord Ciutadà, 2015). En una línea muy similar, Podemos articula, mediante su "Plan de Rescate Ciudadano", los mismos valores: a) el empoderamiento cívico, b) la participación de la ciudadanía, c) la rendición de cuentas y d) la transparencia, con lo que se alinea con las tesis del republicanismo cívico (Podemos, 2015).

La concepción ideológica-programática de Compromís fusiona los elementos regeneradores del sistema democrático con el territorio y la identidad valenciana; esta fusión del repertorio regenerador e identitario es lo que marca las distancias respecto a Podemos y EUPV. Compromís une la existencia de una conciencia nacionalista valenciana a elementos de regeneración democrática, al fomento de la participación y de empoderamiento ciudadano, junto a la defensa de causas de colectivos como los de: LGBTI, ecologistas y feministas, entre otros.

Por tanto, promueven un nacionalismo cívico con tintes comunitaristas y se puede afirmar que esta formación se ha convertido en el principal actor que aglutina la mayoría de votantes con una concepción cultural nacionalista que abarca el fusterianismo, la tercera y cuarta vía, y a parte del autonomismo (Català, 2014; Compromís, 2015; Mezquida, 2015). ${ }^{11}$ De esta forma, Compromís opera como un partido "atrapalotodo" en el clivaje

11 Es lo que se ha llamado Valencianismo de Mayoría por Iniciativa del Poble Valencià. 
nacional valenciano ${ }^{12} \mathrm{y}$, aunque respetan y adoptan los símbolos propios de la autonomía valenciana, no renuncian a transformar el territorio en el "País Valencià." ${ }^{13}$

La diferencia entre Podemos y EUPV en esta cuestión es que ambos promueven parte de los elementos que defiende Compromís, a través de mecanismos plurinacionales (Podemos) o del federalismo (EUPV). No obstante, y a diferencia de formaciones como el PPCV y Ciudadanos, no son beligerantes con Compromís en el tema identitario nacionalista. Lo que sí comparten los tres es un acervo común de valores posmaterialistas (Inglehart, 1991), integrado en un paradigma que aglutina las formas de vida moderna y entretejen una red de redes física y digital.

\section{Conclusiones}

El presente estudio, de carácter exploratorio, se ha centrado en explicar los cambios en el sistema de partidos y en comparar las posiciones actuales de cada uno de los actores principales del sistema de partidos valencianos. Estos últimos se encuentran inmersos en grandes periodos de estabilidad (path dependence) que solo se rompen con la producción de critical junctures, las cuales son aprovechadas a nivel estratégico por estos actores. En dicho escenario se han producido cambios en los niveles de apoyo de estos partidos, lo cual ha derivado en vuelcos electorales que han cambiado el signo político del gobierno (H1a, H1b y H1c).

Tales cambios se han traducido de la siguiente forma en cada uno de los actores estudiados:

12 En la Coalición Compromís encontramos en la actualidad (julio de 2017) la defensa de un nacionalismo netamente valenciano, junto a un nacionalismo cívico integrador, que ha ampliado su base electoral. Asimismo, existe también una minoría alineada con las tesis catalanistas.

13 Los símbolos identitarios alineados con la denominación de País Valencià son: a) la senyera de cuatro barras (el pendón de la conquista); b) la Muixeranga, como el Himno valenciano; c) la unidad de la lengua y el uso habitual de la denominación País Valencià; d) el refuerzo de los vínculos económicos, históricos y culturales entre el País Valencià, Cataluña, las Islas Baleares, Cataluña Norte y la franja de Aragón; e) el fortalecimiento del dinamismo e influencia de una de las regiones más potentes del sur de Europa: la Antigua Corona de Aragón y el Arco Mediterráneo (zona Euram); f) el apoyo a instrumentos que contribuyan a la vertebración del País Valencià ; g) la comarcalización; y h) la recuperación de la memoria histórica. 
1) El PPCV es un partido que, desde que ganó las elecciones a Cortes Valencianas en 1995, ha utilizado las instituciones públicas para perpetuarse en el poder. No es de extrañar que su estrategia sea la de un partido cártel puro que defiende a ultranza los aspectos configuradores del régimen del $78 \mathrm{y}$ la profesionalización de la política. A esta actitud solo hay un "pero": todavía no reconoce el consenso al cual se llegó en 1982 en cuanto a los símbolos de identidad y utiliza este conflicto para atacar a sus rivales políticos. Los populares prefieren centrarse en asuntos como la eficacia de la acción de gobierno antes que en la ética pública y en la defensa de España y su identidad por encima de cualquier pluralismo. Por lo tanto, podemos afirmar que el PPCV es un partido que encarna los rasgos fundamentales del partido cártel.

2) El PSPV-PSOE y Ciudadanos son partidos que se mueven a medio camino entre las estrategias cártel y poscártel, ya que, por una parte, defienden los consensos básicos de la sociedad valenciana, como es la creación de la autonomía y su desempeño; pero, por otra, abogan por cambios cualificados del entramado institucional, a través de la mejora de la representatividad en la transformación de votos en escaños, el fortalecimiento de los mecanismos de rendición de cuentas o la participación ciudadana. El PSPV-PSOE ha sido un partido que ha pasado de utilizar una estrategia cártel a convertirse en un actor reformista del sistema.

Por su parte, Ciudadanos ha devenido en un partido que utiliza una estrategia poscártel en cuanto al componente identitario, pero que se enraíza en una conciencia propia de un partido cártel similar a la del PPCV respecto a la idea de España. Por consiguiente, se puede afirmar que tanto socialistas como Ciudadanos son partidos que combinan estrategias mixtas de cártel y poscártel.

3) Por último, tenemos una serie de actores que abogan por un cambio de sistema desde sus ángulos ideológicos y que asumen las reivindicaciones de la sociedad civil y buscan una ampliación del espacio público. Es el caso de EUPV, Compromís y Podemos que, desde su idea propia del territorio y sus significados, tratan de producir un empoderamiento ciudadano a través de instrumentos de participación ciudadana, del fomento de la transparencia y de la mejora de la rendición de cuentas. Dichos partidos son en mayor o menor medida partidos poscártel y de orientación posmaterialista.

\section{Futuras investigaciones}

Llegados a este punto y tras exponer las dos variables señaladas - cartelización/poscartelización y el componente identitario-, consideramos que los 
principales desarrollos más potentes a corto y medio plazo son: a) el análisis del discurso de las formaciones políticas que, desde una serie histórica, permita comprobar los cambios experimentados por cada una de ellas, y b) el diseño de un plan de entrevistas en profundidad con actores políticos relevantes sobre la identidad y las estrategias de los partidos para conocer su evolución real.

Una tercera vía, a largo plazo, aspira a realizar una investigación cuantitativa que posibilite conocer las posturas de afiliados, simpatizantes y votantes de las formaciones actuales, así como los cambios en los perfiles sociodemográficos de los votantes.

\section{Referencias}

Acord Ciutadà (2015), Programa para las elecciones autonómicas 2015. Disponible en: http://servicios.lasprovincias.es/documentos/acord-ciutada-programa-electoralmunicipales-autonomicas-2015.pdf [10 de julio de 2017].

Baumann, Zigmund (2000), Modernidad Liquida, Argentina: Fondo de Cultura Económica. Berger, Peter y Luckmann, Thomas (1975), The Social Construction of Reality. A Theatrise in the Sociology of Knowledge, Inglaterra: Penguin Books.

Bodoque, Anselm (2009), "Unió Valenciana (1982-2008). Una aproximación", en Papers, núm. 92, España: Universidad Autónoma de Barcelona. http://dx.doi.org/10.5565/ rev/papers/v92n0.713.

Brubaker, Rogers (1990), "Immigration, Citizenship, and the Nation-State in France and Germany: A Comparative Historical Analysis", en International Sociology, núm. 5, Reino Unido: Sage. http://dx.doi.org/10.1177/026858090005004003.

Capoccia, Giovanni y Kelemen, Daniel (2007), "The Study of Critical Junctures: Theory, Narrative and Counterfactuals in Historical Institutionalism", en World Politics, vol. 3, núm. 59, Estados Unidos: Cambridge University Press. https://doi.org/10.1017/ S0043887100020852.

Castells, Manuel (1999), La era de la información. Economia, Sociedad y Cultura. Vol. 1. La sociedad Red, España: Alianza.

Català, Lluís (2012), Fonaments de la identitat territorial amb especial atenció a la identitat nacional. El cas valencià: discursos politics sobre la identitat valenciana entre militants de base del Bloc, EUPV i PSPV-PSOE, España: Universidad de Alicante.

Català, Lluís (2014), "Discursos polítics sobre la identitat territorial en els partits valencians d'esquerra i de centreesquerra”, en Papers, núm. 99, España: Universidad Autónoma de Barcelona. http://dx.doi.org/10.5565/rev/papers.613.

Ciudadanos (2015), Programa Electoral Comunidad Valenciana 2015 "100 propuestas para un proyecto de buen gobierno". Disponible en: http://indiceempoderamiento. es/locales2015/wpcontent/uploads/2015/05/Ciudadanos.pdf $[10$ de julio de 2017].

Compromís (2015), \#Convalentía. Programa de gobierno a Corts 2015. Disponible en: http://indiceempoderamiento.es/locales2015/wp-content/uploads/2015/05/ Compromis.pdf [10 de julio de 2017]. 
Della-Porta, Donatella (2002), "Los actores de la corrupción: políticos de negocios en Italia”, en Gestión y análisis de Politicas Públicas, núm. 21, España: Instituto Nacional de Administración Pública. http://dx.doi.org/10.24965/gapp.vi21.261.

Detterbeck, Klaus (2005), "Cartel parties in Western Europe?”, en Party Politics, vol. 2, núm. 11, Inglaterra: Sage. https://doi.org/10.1177/1354068805049738.

DiMaggio, Paul (1988), "Interest and Agency in Institutional Theory", en Zucker, Lynne [ed.], Institutional Patterns and Organizations: culture and environment, Reino Unido: Ballinger.

Downs, Anthony (1957), "An economic theory of political action in a democracy", en Journal of Political Economy, vol. 2, núm. 65, Estados Unidos: University of Chicago Press. https://doi.org/10.1086/257897.

Duverger, Maurice (1957), Los partidos politicos, México: Fondo de Cultura Económica.

Easton, David (1997), "The future of the post-behavioral phase in Political Science”, en Monroe, Kristen [ed.], Contemporary empirical political theory, Estados Unidos: University of California Press.

Franch, Vicent (1995), "Las elecciones autonómicas valencianas en perspectiva (19831991): los efectos de la cláusula de barrera del $5 \%$ en el sistema de partidos valenciano", en Cuadernos constitucionales de la Cátedra Fadrique Furió Ceriol, núm. 9-10, España: Universitat de València.

Franch, Vicent (1996), "Las elecciones del cambio: las autonómicas y municipales del 28 de mayo de 1995 en la 'Comunitat Valenciana”, en Revista Valenciana d'Estudis Autonòmics, núm. 15, España: Generalitat Valenciana.

Franch Vicent (1998), “Las elecciones autonómicas en la Comunitat Valenciana”, en Alcántara, Manuel y Martínez, Antonia [coords.], Las elecciones autonómicas en España, 1980-1997, España: Centro de Investigaciones Sociales.

Franch, Vicent y Hernández, Juan (2005), “El subsistema de partidos valenciano después de las elecciones autonómicas del 25 de mayo de 2003”, en Franch, Vicent [ed.], Elecciones autonómicas y municipales de 2003 en la Comunitat Valenciana, España: Generalitat Valenciana.

Franch, Vicent y Martín-Cubas, Joaquín (1999), "El comportamiento electoral de los valencianos: continuidad y cambio en las tres citas electorales del 13 de junio de 1999”, en Revista Valenciana d'Estudis Autonòmics, núm. 29, España: Generalitat Valenciana.

Franklin, Mark (1992), “The decline of cleavage politics”, en Franklin, Mark et al. [eds.], Electoral Change, Reino Unido: Cambridge University Press.

Fuster, Joan (1967), Nosaltres, els valencians, España: Edicions 62.

Garcia-Oliver, Ferran (2016), Valencians sense ADN. El relat dels origins, España: Tres i Quatre.

Hernàndez i Martí, Gil y Albert, María (2012), "La dinámica general de la política cultural en el País Valenciano: posiciones, discursos y prácticas de los actores culturales valencianos", en Revista de Investigaciones Políticas y Sociológicas, núm. 11, España: Universidad de Santiago de Compostela.

Hutcheson, Dereck (2012), "Party cartels beyond Western Europe: Evidence from Russia”, en Party Politics, vol. 6, núm. 19, Inglaterra: Sage. https://doi. org/10.1177/1354068811436033.

Immergut, Ellen (1998), “The theoretical core of the new institutionalism”, en Politics and Society, vol. 26, núm. 1, Estados Unidos: Sage. https://doi.org/10.1177/0032329298 026001002. 
Inglehart, Ronald (1991), El cambio cultural, España: Centro de Investigaciones Sociológicas.

Katz, Richard y Mair, Peter (2012), "La supremacía del partido en las instituciones públicas: el cambio organizativo de los partidos en las democracias contemporáneas”, en Delgado, Irene y López-Nieto, Lourdes, Doce lecturas fundamentales de Ciencia Política, España: Universidad Nacional de Educación a Distancia.

Kirchheimer, Otto (1966), "The Transformation of the Western European Party System”, en LaPalombara, Joseph y Weiner, Myron [eds.], Political Parties and Political Development, Inglaterra: University Press.

Kitschelt, Herbert (2004), "Diversificación y reconfiguración de los sistemas de partidos de las democracias postindustriales", en Revista Española de Ciencia Política, núm. 10, España: Asociación Española de Ciencia Política.

Kopecky, Petr y Mair, Peter (2011), "Party patronaje as an Organizational Resource", en Kopecky, Petr et al. [eds.], Party Patronage and Party Government in European Democracies, Reino Unido: Oxford University Press.

Landman, Todd (2011), Politica Comparada, España: Alianza.

López-Nieto, Lourdes (2010), “Notas sobre la hipotética 'cartelización' de los partidos en el estado autonómico”, en Arenilla, Manuel [coord.], La Administración Pública entre dos siglos, España: Instituto Nacional de Administración Pública.

Mahoney, James (2000), "Path Dependence in Historical Sociology", en Theory and Society, núm. 29, Países Bajos: Springer. https://doi.org/10.1023/A:1007113830879.

Mahoney, James y Thelen, Kathleen (2010), Explaining Institutional Change: Ambiguity, Agency and Power, Inglaterra: Cambridge University Press.

Manin, Bernard (1999), Los Principios del Gobierno Representativo, España: Alianza.

Martín-Cubas, Joaquín (2007), La polémica identidad de los valencianos: a propósito de las reformas de los Estatutos de Autonomia. Estudio/WorkingPaper 2007/258, España: Universitat Autònoma de Barcelona.

Martín-Cubas, Joaquín (2015), "La ruptura de un ciclo electoral en la Comunidad Valenciana: estudio comparado de los resultados de las elecciones locales y autonómicas de 2015", en Participació Electoral $i$ Territori. Análisi de les eleccions municipals $i$ autonómiques 2015, España: Universitat de València.

Martínez-Sospedra, Manuel (1996), "Participación política, autonomía y ley electoral: la ley electoral valenciana”, en Corts: Anuario de Derecho Parlamentario, núm. 2, España: Cortes Valencianas.

Martínez-Sospedra, Manuel (1999), "Bajo el signo del centro-derecha: ¿recambio en el sistema de partido dominante?”, en Revista Valenciana d'Estudis Autonómics, núm. 29, España: Generalitat Valenciana.

Mezquida, Amadeu (2015), El valencianisme enfront d'Espanya, España: Fundació Nexe.

Mira, Joan (2015), La Nació dels Valencians, España: Raval Edicions.

Oliver, Christine (1988), “The Collective Strategy Framework: An Application to Competing Predictions of Isomorphism”, en Administrative Science Quarterly, vol. 33, núm. 4, Estados Unidos: Cornell University. https://doi.org/10.2307/2392643.

Oñate, Pablo (2013), "Los partidos políticos y los sistemas de partidos en la Comunidad Valenciana”, en Canales, José y Menéndez, Manuel [eds.], El sistema político y administrativo valenciano, España: Tirant Lo Blanch.

Pardo, Gonzalo y Abellán, María Ângeles (2016), "La dinámica del sistema político-electoral valenciano: una aproximación a sus principales transformaciones (2003-2015)", 
en Santos-González, Diego y Giménez-Rodríguez, Silvia [coord.], Integraciones y desintegraciones sociales: Pobreza, migraciones, refugio, España: Asociación CastellanoManchega de Sociología.

Pardo, Gonzalo y Abellán, María Ángeles (2017), "El proceso de cartelización en entornos institucionalizados: legitimación y comunicación política", en Miguel Hernández Communication Journal, núm. 8, España: Universidad Miguel Hernández.

Peters, Guy (2000), Institutional Theory: Problems and Prospects. Working paper of the Institute for Advanced Studies, Austria: Institute for Advance Studies.

Peters, Guy (2003), Institutional Theory in Political Science. The New Institutionalism, Inglaterra: Pinter.

Pfeffer, Jeffrey y Salancik, Gerald (1978), The External Control of Organizations. A Resource Dependence Perspective, Estados Unidos: Stanford University Press.

Pierson, Paul (2004), Politics in Time. History, Institutions and Social Analysis, Estados Unidos: Princeton University Press.

Podemos (2015), El programa de la Comunidad Valenciana. Disponible en: http:// indiceempoderamiento.es/locales2015/wp-content/uploads/2015/05/Podemos.pdf [10 de julio de 2017].

PPCV (2015), Elecciones autonómicas mayo 2015. Programa de Gobierno Comunitat Valenciana. Disponible en: http://indiceempoderamiento.es/locales2015/wpcontent/uploads/2015/05/PP.pdf [10 de julio de 2017].

PSPV-PSOE (2015), 2015 elecciones autonómicas. Disponible en: http://www.lasprovincias. es/elecciones/autonomicas-municipales/201505/11/programa-electoral-psoecomunitat-20150511125934.html [10 de julio de 2017].

PSPV-PSOE (2015a), Un gobierno comprometido con la ciudadania. Medidas del programa electoral. Disponible en: http://servicios.lasprovincias.es/documentos/pspv-medidasconcretas-municipales-autonomicas-2015.pdf [10 de julio de 2017].

Reif, Karlheinz y Schmitt, Hermann (1980), "Nine Second-order national elections-a conceptual framework for the analysis of european election results", en European Journal of Political Research, vol. 1, núm. 8, Estados Unidos: Wiley. https://doi. org/10.1111/j.1475-6765.1980.tb00737.x.

Sandri, Giulia y Pauwels, Teun (2010), "Party Membership Role and Party Cartelization in Belgium and Italy: Two Faces of the Same Medal?", en Politics \& Policy, vol. 38, núm. 6, México: Policy Studies Organization y Universidad Iberoamericana. http://dx.doi. org/10.1111/j.1747-1346.2010.00276.x.

Sanmartín, José (2010), La Comunitat Valenciana en el segle XXI. Reptes i solucions, España: Editorial Club Universitario.

Sanz-Díez, Jesús (1982), La cara secreta de la política valenciana. De la predemocracia al Estatuto de Benicassim, España: F. Torres.

Sanz-Díez, Benito y Felip i Sardà, Josep Maria (2006), La construcción política de la Comunitat Valenciana, 1962-1982, España: Alfons El Magnànim.

Sartori, Giovanni (2005), Parties and Party Systems. A framework for analysis, Inglaterra: European Consortium of Political Research.

Steinmo, Sven (2013), "Institucionalismo Histórico", en Della Porta, Donatella y Keating, Michael [eds.], Enfoques y metodologias de las Ciencias Sociales, España: Akal.

Thelen, Kathleen (2003), "How Institutions Evolve: Insights from Comparative Historical Analysis", en Mahoney, James y Rueschemeyer, Dietrich [eds.], Comparative Historical Analysis in the Social Sciences, Inglaterra: Cambridge University Press. 
Vallès i Sanchis, Ismael (2000), "Nacionalismes al País Valencià: una proposta de tipologies des de la geografía política”, en Cuadernos de Geografía, núm. 65-66, España: Universidad de Valencia.

van der Eijk, Cees et al. (1992), "Cleavages, conflict resolutions and democracy", en Franklin, Mark et al. [eds.], Electoral Change, Inglaterra: Cambridge University Press.

Yishai, Yael (2001), "Bringing Society Back in Post-Cartel Parties in Israel", en Party Politics, vol. 6, núm. 7, Reino Unido: Sage. https://doi.org/10.1177/1354068801007006001.

\title{
Anexo
}

Tabla 1

\section{Caracterización de las estrategias cártel y poscártel}

\author{
Estrategia cártel Estrategia poscártel
}

a) La definición según sus intereses en aspectos capitales del sistema electoral y el sistema de financiación.

b) El establecimiento de estrategias de cooptación basadas en el patronazgo (Kopecky y Mair, 2012).

c) El uso de recursos del Estado para la colocación de partidarios en puestos de responsabilidad.

d) La articulación de estrategias y mensajes comunicativos con una tendencia centrípeta entre el partido del gobierno y el partido de la oposición.

e) El uso de los resortes comunicativos propios de un sistema de pluralismo polarizado para marcar la agenda comunicativa (Pardo y Abellán, 2017). a) La recuperación de la relación del partido con la sociedad civil a través de:

- la asunción de sus demandas como propias y la defensa de causas demandadas por la sociedad.

- la recaptación de fondos y apoyo a su acción por parte de la sociedad civil.

- el uso de recursos humanos para puestos de responsabilidad.

b) La inclusión de fracturas que se producen en la sociedad que dependen del sentimiento de pertenencia.

c) La construcción de una alternativa en contra de los partidos cártel, a través de cambios profundos en la legislación constitutiva, las estructuras institucionales y los aspectos funcionales.

d) La utilización de los nuevos medios de comunicación y su bidireccionalidad simétrica y simultánea para marcar la agenda comunicativa.

Fuente: Elaboración propia. 


\section{Tabla 2}

Estrategias cártel y out cártel en el periodo 1978-1982

\begin{tabular}{cc}
\hline Partidos dentro del cártel & Partidos fuera del cártel \\
\hline & PSAN \\
PSPV-PSOE & PSPV \\
& PSOE-PV \\
& PCE \\
UCD-RV & UDPV \\
& PNPV \\
& UCD-RV \\
URV \\
FN \\
AP
\end{tabular}

Fuente: Elaboración propia.

Tabla 3

El posicionamiento partidista sobre la identidad valenciana desde la transición hasta 1982 (etapa preautonómica)

\begin{tabular}{ccc}
\hline $\begin{array}{c}\text { Catalanismo } \\
\text { (Fusterismo })\end{array}$ & Identidad estatutaria & Regionalismo o Centralismo \\
\hline PSAN & UCD-RV & UCD-RV \\
MCPV & URV \\
PSPV & PSPV-PSOE & FN \\
PSOE-PV & PSOE-PV & AP \\
PCE & \\
UDPV & PCPV-PCE & \\
PNPV & & \\
\hline
\end{tabular}

Fuente: Adaptado de Martín-Cubas (2007). 


\section{Tabla 4}

Estrategias cártel y out cártel en el periodo 1982-2015

\begin{tabular}{cc}
\hline Partidos dentro del cártel & Partidos fuera del cártel \\
\hline PP & AP/CP \\
& PPCV \\
& UV \\
& CV \\
& PSAN \\
& ERC-ERPV \\
& PNV \\
& UDPV \\
& PNV \\
& UDPV \\
& BNV \\
& Compromís \\
\end{tabular}

Fuente: Elaboración propia.

Tabla 5

\section{El posicionamiento de los partidos políticos respecto a la identidad estatuaria 1995-2015}

\begin{tabular}{cccc}
\hline $\begin{array}{c}\text { Catalanismo } \\
\text { (Fusterismo) }\end{array}$ & Tercera Vía & $\begin{array}{c}\text { Identidad } \\
\text { estatutaria }\end{array}$ & $\begin{array}{c}\text { Regionalismo o } \\
\text { Centralismo }\end{array}$ \\
\hline PSAN & PNV & & AP/CP \\
ERC-ERPV & UDPV & PPCV & PPCV \\
EUPV & & PSPV-PSOE & UV $\downarrow$ \\
$\mathrm{UPV} \longrightarrow$ & BNV & PCPV/EU & CV $\downarrow$ \\
& Compromís & & \\
\hline
\end{tabular}

Fuente: Adaptado de Martín-Cubas (2007). 
Tabla 6

Estrategias cártel y out cártel en la actualidad

\begin{tabular}{cc}
\hline Partidos dentro del cártel & Partidos fuera del cártel \\
\hline PPPV-PSOE & CV \\
Compromís & PSAN \\
& Compromís \\
& EU-Acord Ciutadà \\
& Podemos \\
& Ciudadanos \\
& ERC-ERPV \\
\hline
\end{tabular}

Fuente: Elaboración

María Ángeles Abellán-López. Doctora en Derecho por la Universidad de Alicante y profesora de Ciencia Política y de la Administración en la Universidad Miguel Hernández de Elche (España). Principales líneas de investigación: la rendición de cuentas de la gestión pública y la calidad de la democracia, el buen gobierno y la educación ética-cívica con perspectiva de género. Publicaciones recientes: "El origen y la evolución de la Sindicatura de Comptes de la Comunitat Valenciana 1985-2015", en Revista de Contabilidad y Tributación. Centro de Estudios Financieros, núm. 418, España (2018); "Del cielo normativo a la tosca materia. La transformación discursiva del buen gobierno en gobernanza", en Foro Interno: Anuario de Teoría Política, vol. 17, España (2017); "Los órganos de control externo (OCEX): una aproximación desde la perspectiva de las Relaciones Intergubernamentales", en Revista Vasca de Administración Pública, núm. 109, España (2017); "Las políticas públicas simbólicas para el buen gobierno y la calidad democrática en la Comunitat Valenciana”, en Inguruak. Revista Vasca de Sociología y Ciencia Política, núm. 62, España (2017). 
Gonzalo Pardo-Beneyto. Licenciado en Ciencias Políticas y de la Administración por la Universidad Miguel Hernández y Máster Universitario en Política y Democracia por la Universidad Nacional de Educación a Distancia (UNED). En la actualidad compagina sus estudios como Doctorando en Derecho con actividades de consultoría e impartiendo clase en el Instituto Mediterráneo de Estudios de Protocolo-Centro Adscrito a la Universidad Miguel Hernández, España. Principales líneas de investigación: procesos de innovación y modernización en las administraciones públicas y aspectos centrados en la calidad democrática, el buen gobierno y la participación ciudadana. Publicaciones recientes: "Las políticas públicas simbólicas para el buen gobierno y la calidad democrática en la Comunitat Valenciana”, en Inguruak. Revista Vasca de Sociología y Ciencia Politica, núm. 62, España, (2017); "El proceso de cartelización en entornos institucionalizados: legitimación y comunicación política”, en Miguel Hernández Communication Journal, núm. 8, España (2017); "La integración necesaria entre los enfoques de gestión pública y los procesos participativos democráticos. El caso de \#ontinyentparticipa”, en Revista Enfoques: Ciencia Politica y Administración Pública, núm. 24, vol. 14, Chile (2016); "El uso de las cartas de servicio en la administración local: el caso del Ayuntamiento de Torrent", en Barataria: revista castellano-manchega de ciencias sociales, España (2016).

Recepción: 10 de noviembre de 2017.

Aprobación: 21 de marzo de 2018. 